\title{
Acúmulo de matéria seca e produtividade em híbridos de arroz irrigado simulados com o modelo SimulArroz
}

\author{
Giovana Ghisleni Ribas (1), Nereu Augusto Streck(1), Isabel Lago(1), Alencar Junior Zanon (2), \\ Daniel Arthur Gaklik Waldow ${ }^{(2)}$, Ary José Duarte Junior ${ }^{(1)}$, \\ Moisés de Freitas do Nascimento(1) e Vanessa Fontana ${ }^{(3)}$
}

\begin{abstract}
(1)Universidade Federal de Santa Maria (UFSM), Avenida Roraima, no 1000, Cidade Universitária, Prédio 77, Sala 2, Camobi, CEP 97105-900 Santa Maria, RS, Brasil. E-mail: giovana.ghisleni@hotmail.com, nstreck2@yahoo.com.br, isalago08@yahoo.com.br, ary.duartee@gmail.com, moises.fn@gmail.com (2)Instituto Rio Grandense do Arroz Irrigado, Avenida Missões, no 342, São Geraldo, CEP 90230-100 Porto Alegre, RS, Brasil. E-mail: alencarzanon@hotmail.com, daniel-waldow@irga.rs.gov.br (3)Universidade Luterana do Brasil, Avenida Farroupilha, no 8001, São José, CEP 92425-900 Canoas, RS, Brasil. E-mail: wanessafontana@yahoo.com.br
\end{abstract}

Resumo - O objetivo deste trabalho foi avaliar a calibração e o desempenho do modelo SimulArroz na simulação do acúmulo de matéria seca e da produtividade de grãos de cultivares de arroz híbrido irrigado por inundação no Rio Grande do Sul. Os experimentos foram conduzidos em delineamento de blocos ao acaso em Santa Maria, Cachoeirinha, Santa Vitória do Palmar, Uruguaiana, Bagé, Camaquã e Cachoeira do Sul. Em Santa Maria e Cachoeirinha, foram avaliados o acúmulo de matéria seca da parte aérea e a produtividade de grãos de três cultivares híbridas (Prime CL, Inov CL e QM 1010 CL) e uma convencional de arroz usada como testemunha (Irga 424). Nos outros locais, foi avaliada somente a produtividade de grãos. Observou-se que, nas cultivares híbridas, são alocados mais fotoassimilados da parte aérea nos colmos, para sustentar a maior panícula, em comparação às cultivares convencionais de arroz. A calibração do modelo SimulArroz utilizada é eficiente em simular o acúmulo de matéria seca e a produtividade de grãos de cultivares de arroz híbrido nas regiões orizícolas irrigadas do Rio Grande do Sul.

Termos para indexação: Oryza sativa, crescimento, partição de matéria seca, rendimento.

\section{Accumulated dry matter and grain yield in flooded hybrid rice simulated with the SimulArroz model}

\begin{abstract}
The objective of this work was to evaluate the calibration and the performance of the SimulArroz model for simulating the accumulated dry matter and the grain yield of flooded hybrid rice cultivars in the state of Rio Grande do Sul, Brazil. The experiments were carried out in a completely randomized block design in the municipalities of Santa Maria, Cachoeirinha, Santa Vitória do Palmar, Uruguaiana, Bagé, Camaquã, and Cachoeira do Sul. In Santa Maria and Cachoeirinha, the shoot dry matter accumulation and grain yield of three hybrid cultivars (Prime CL, Inov CL, and QM 1010 CL) and one conventional rice cultivar, used as control (Irga 424), were evaluated. In the other sites, only grain yield was evaluated. It was observed that more photoassimilates from the culm shoots are allocated in the hybrid cultivars, in order to sustain a greater panicle, compared with conventional rice. The calibration of the SimulArroz model used is efficient to simulate the accumulated dry matter and grain yield of hybrid rice cultivars in flooded rice crop regions of the state of Rio Grande do Sul.
\end{abstract}

Index terms: Oryza sativa, growth, dry matter partitioning, yield.

\section{Introdução}

$\mathrm{O}$ arroz é o segundo cereal mais produzido no mundo, e o Brasil é o nono maior produtor mundial (Usda, 2016). A região Sul do Brasil contribui, em média, com $76 \%$ da produção nacional, e no Rio Grande do Sul são cultivados anualmente 1,1 milhão de hectares com arroz (Conab, 2016) - este Estado é responsável por $67 \%$ da produção nacional (Irga, 2016a). Na área agrícola, modelos matemáticos são cada vez mais utilizados em diversas culturas, como a soja, o milho, a mandioca, e o arroz, entre outras, para descrever as interações genótipo-ambiente (Sentelhas et al., 2015). No Brasil, o uso de modelos de simulação de crescimento, desenvolvimento e produtividade das culturas agrícolas aumentou nos últimos anos (Li et al., 
2013; Gabriel et al., 2014; Rosa et al., 2015; Sentelhas et al., 2015). Com base nos modelos matemáticos, é possível realizar estudos de respostas em crescimento, desenvolvimento e produtividade de grãos em cenários de mudança climática, previsão de safra, e também estimativa da data de ocorrência de estádios chaves de desenvolvimento que estão associados a práticas de manejo (Torrion et al., 2011; Dingkuhn et al., 2015; Grassini et al., 2015). Um destes é o SimulArroz, que simula o crescimento, o desenvolvimento e a produtividade de grãos na cultura do arroz, e foi desenvolvido para cultivares usadas no sistema de irrigação por inundação no Rio Grande do Sul (Streck et al., 2011; Rosa et al., 2015). O SimulArroz calcula os processos de crescimento por meio do acúmulo de matéria seca nos diferentes órgãos da planta que fundamentalmente determinam a produtividade da cultura (Rosa et al., 2015), e já foi utilizado para estudos do impacto da mudança climática, projetada para este século, sobre a cultura do arroz no Rio Grande do Sul, com a versão 1.0 do modelo que tem apenas cultivares convencionais (Walter et al., 2014). A principal diferença entre o arroz convencional e o arroz híbrido é a heterose, que se expressa em caracteres quantitativos como produtividade de grãos, peso de grãos, estatura de plantas, entre outros, o que permite atingir produtividades 20 a $30 \%$ maiores que nas cultivares convencionais (Chin et al., 2012; Coimbra et al., 2006). Porém, na atual versão do SimulArroz (versão 1.0), não há a opção de simulação para cultivares híbridas de arroz, o que é uma limitação, pois no Rio Grande do Sul o arroz híbrido é cultivado desde 2002, em uma área de aproximadamente $68 \mathrm{mil}$ hectares (Irga, 2015), com perspectivas de aumentar seu cultivo nos próximos anos.

O objetivo deste trabalho foi avaliar a calibração e o desempenho do modelo SimulArroz na simulação do acúmulo de matéria seca e da produtividade de grãos de cultivares de arroz híbrido irrigado por inundação no Rio Grande do Sul.

\section{Material e Métodos}

Foram conduzidos experimentos de campo durante o ano agrícola 2013/2014, em Santa Maria $\left(29^{\circ} 72^{\prime} \mathrm{S}, 53^{\circ} 72^{\prime} \mathrm{W}\right.$ e altitude de $\left.103 \mathrm{~m}\right)$, e no ano agrícola 2014/2015, em Santa Maria e nas estações de pesquisa do Instituto Rio Grandense do Arroz
(Irga) de Cachoeirinha $\left(29^{\circ} 57^{\prime} \mathrm{S}, 51^{\circ} 5^{\prime} \mathrm{W}\right.$ e $17 \mathrm{~m}$ de altitude), Santa Vitória do Palmar ( $33^{\circ} 51^{\prime} \mathrm{S}, 53^{\circ} 35^{\prime} \mathrm{W}$ e $24 \mathrm{~m}$ de altitude), Uruguaiana $\left(29^{\circ} 83^{\prime} \mathrm{S}, 57^{\circ} 08^{\prime} \mathrm{W}\right.$ e $74 \mathrm{~m}$ de altitude), Bagé $\left(31^{\circ} 34^{\prime} \mathrm{S}, 54^{\circ} 01^{\prime} \mathrm{W}\right.$ e $226 \mathrm{~m}$ de altitude), Camaquã ( $30^{\circ} 80^{\prime} \mathrm{S}, 51^{\circ} 83^{\prime} \mathrm{W}$ e $92 \mathrm{~m}$ de altitude) e Cachoeira do $\mathrm{Sul}\left(30^{\circ} 02^{\prime} \mathrm{S}, 52^{\circ} 53^{\prime} \mathrm{W}\right.$ e $68 \mathrm{~m}$ de altitude). Estes sete locais representam as condições edafoclimáticas da área de cultivo de arroz irrigado no Rio Grande do Sul.

As regiões da Campanha e Fronteira Oeste, representadas pelos municípios de Bagé e Uruguaiana, apresentam alta disponibilidade de radiação solar nos meses de dezembro, janeiro e fevereiro $(27,0,25,0$ e 21,9 $\mathrm{MJ} \mathrm{m}^{-2}$ dia $^{-1}$ em Bagé; e 26,39, 26,13 e 23,20 $\mathrm{MJ} \mathrm{m}^{-2} \mathrm{dia}^{-1} \mathrm{em}$ Uruguaiana). Uruguaiana apresenta as temperaturas mínimas $\left(18,6,19,7\right.$ e $\left.19,6^{\circ} \mathrm{C}\right)$ e máximas $\left(31,2,31,8\right.$ e $\left.31,2^{\circ} \mathrm{C}\right)$ mais elevadas neste mesmo período, enquanto Bagé tem as menores temperaturas mínimas do Estado nos meses de setembro a dezembro (11,0, $12,6,14,7$ e $\left.17,0^{\circ} \mathrm{C}\right)$, o que acarreta menor temperatura do solo e atraso do período recomendado de semeadura (Irga, 2016a). A região Depressão Central, representada pelos municípios de Santa Maria e Cachoeira do Sul, juntamente com a Fronteira Oeste, apresentam as maiores temperaturas máximas nos meses de dezembro, janeiro e fevereiro $\left(19,1,30\right.$ e $\left.29,5^{\circ} \mathrm{C}\right)$. A Planície Costeira Interna e a região da Zona Sul são representadas pelos municípios de Cachoeirinha, Camaquã e Santa Vitória do Palmar, com as menores probabilidades de ocorrência de temperatura igual ou superior a $35^{\circ} \mathrm{C}$ nos meses de dezembro, janeiro e fevereiro (Mota, 1995), e o Município de Santa Vitória do Palmar tem alto risco de esterilidade em razão das baixas temperaturas nos meses de dezembro, janeiro e fevereiro, em que as temperaturas mínimas são 17,3, 18,9 e $19,2^{\circ} \mathrm{C}$, respectivamente.

Foram utilizadas as cultivares de arroz híbrido Prime CL e Inov CL, de ciclo Precoce; a QM 1010 CL, de ciclo médio; e a cultivar convencional Irga 424 (testemunha). A cultivar convencional foi escolhida para comparação, pois é indicada para todas as regiões orizícolas do Rio Grande do Sul, onde é comum a ocorrência de temperaturas mais baixas; além disso, tem a maior produtividade potencial, aceitável qualidade de grãos e ocupa a maior área plantada no estado entre as cultivares convencionais não CL - "Clearfield system" - (Reunião..., 2014). O espaçamento entre linhas foi de $0,17 \mathrm{~m}$ em todos os locais, e a área de cada parcela 
e o tipo de delineamento utilizado estão descritos na Tabela 1.

As amostragens de plantas para avaliação do acúmulo de massa de matéria seca (MS) da parte aérea, em Santa Maria e Cachoeirinha, foram realizadas conforme o método utilizado por Gabriel et al. (2014) (Tabela 1). Assim, as parcelas foram divididas em quadrantes, e em cada uma foram cortadas, ao nível do solo, e coletadas as plantas de 3 linhas de $1,0 \mathrm{~m}$ de comprimento $\left(0,51 \mathrm{~m}^{2}\right)$. Após a coleta, foi realizada a separação em folhas verdes, folhas senescentes, colmos e panículas, que foram levadas para a secagem em estufa sob ventilação forçada a $60^{\circ} \mathrm{C}$ até a estabilização do peso do material e pesagem em balança de precisão $(0,001 \mathrm{~g})$. A produtividade de grãos foi obtida com a colheita de grãos em uma área de $5 \mathrm{~m}^{2}$ nos Valores de Cultivo e Uso (VCUs). Nos outros experimentos de campo (Santa Maria e Cachoeirinha), a produtividade de grãos (Prod) foi estimada com base nos componentes de produtividade, conforme a equação: Prod $=\mathrm{NP} \times \mathrm{NGP} \times(\mathrm{PMG} / 1.000)$, em que NP é o número de panículas $\mathrm{m}^{-2}$, NGP é o número de grãos por panícula e o PMG é o peso de mil grãos em gramas. A produtividade foi convertida para $\mathrm{Mg} \mathrm{ha}^{-1}$.

A estimativa da produtividade foi realizada com a contagem do número de panículas em $1 \mathrm{~m}^{2}$ e a coleta de 15 panículas aleatoriamente, para a contagem do número de grãos por panícula. Posteriormente, foram amostrados mil grãos, tendo-se descartado os abortados e os com danos ocasionados pelo ataque de pragas ou doenças, pois a calibração do modelo é na condição potencial. O material foi seco em estufa de circulação forçada até o peso constante ( 72 horas). Com base nos componentes de produtividade, foi

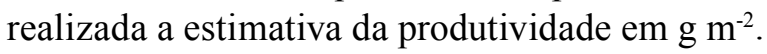

A versão 1.0 do SimulArroz (Universidade Federal de Santa Maria, Santa Maria, RS, Brasil) foi usada neste estudo. A produção diária de matéria seca no modelo SimulArroz é calculada com base na eficiência do uso da radiação (EUR) e do índice de área foliar (IAF). A EUR é calculada pela equação: $\mathrm{EUR}=2,39 \times \mathrm{FC} \times \mathrm{f}\left(\mathrm{CO}_{2}\right) \times \mathrm{f}(\mathrm{NT})$, em que FC é o fator de correção da EUR em função da temperatura média do ar, $\mathrm{f}\left(\mathrm{CO}_{2}\right)$ é a função resposta ao $\mathrm{CO}_{2}, \mathrm{f}(\mathrm{NT})$ é a função de correção do nível tecnológico da lavoura, e 2,39 é o valor da EUR para as cultivares convencionais de arroz na versão 1.0 do SimulArroz (Kiniry et al., 2001). O efeito da temperatura sobre a EUR é descrito com uma função de resposta com quatro temperaturas cardinais (Soltani et al., 2001), de modo quea EUR émáxima quando a temperatura média diária do ar se situa na faixa ótima $\left(22\right.$ a $\left.32^{\circ} \mathrm{C}\right)$ e é multiplicada por um fator de correção menor que $1 \mathrm{em}$ temperaturas sub e supra ótimas, que reduz linearmente a EUR até zero, quando a temperatura é igual ou menor que $9^{\circ} \mathrm{C}$ ou igual ou maior que $45^{\circ} \mathrm{C}$. A concentração de $\mathrm{CO}_{2}$ utilizada foi 390 ppm (Streck et al., 2012) e o modelo foi rodado em nível tecnológico potencial. A função resposta que multiplica a EUR na equação anterior é: $\mathrm{f}\left(\mathrm{CO}_{2}\right)=\left[3,096 \times 10^{23}+1,4382\left(\mathrm{CO}_{2}\right)^{8,378}\right] /$ $\left[3,096 \times 10^{23}+\left(\mathrm{CO}_{2}\right)^{8,378}\right]$.

A produção diária de massa de matéria seca no SimulArroz foi calculada segundo Aggarwal et al. (2006): MS = EUR $\times$ RFA (1-EXP(-CER x IAF)) em que MS é a massa de matéria seca $\left(\mathrm{g} \mathrm{m}^{-2} \mathrm{dia}^{-1}\right)$, EUR é a eficiência do uso da radiação $\left(\mathrm{g} \mathrm{MJ}^{-1}\right)$, RFA é a radiação fotossinteticamente ativa, considerada $50 \%$ da radiação solar global incidente $\left(\mathrm{MJ} \mathrm{m}^{-2} \mathrm{dia}^{-1}\right)$, a qual foi calculada com base no brilho solar pela equação de Angstron, modificada por Prescott e Penman (Estefanel et al., 1990), e CER é o coeficiente de extinção da radiação no dossel, considerado como 0,4 até a antese e 0,6 após a antese (Bouman et al., 2004).

Depois de calculada a produção de MS total diária, o SimulArroz calcula a partição da MS total entre raiz e parte aérea, e a fração da parte aérea é distribuída entre os diferentes órgãos da planta (folhas, colmos, folhas senescentes, panícula e grãos) por meio de frações (entre 0 e 1) em uma tabela de contingência e conforme o estádio de desenvolvimento em que a cultura se encontra, segundo a escala de Counce et al. (2000). O valor atual utilizado pelo modelo SimulArroz para o fator de formação de espiguetas (SOCF) é de 65 espiguetas por gMS - este valor pode sofrer variação dependendo da cultivar, como, por exemplo, as cultivares híbridas. Dessa maneira, o valor de 65 espiguetas por gMS foi calibrado para os híbridos com o objetivo de obter melhor ajuste na produtividade. $\mathrm{O}$ método utilizado para fazer esse ajuste foi o de aumento e diminuição de um valor inicial em $1 \%$ até minimizar o erro da estimativa (Samboranha et al., 2013).

A produtividade de grãos $\left(\mathrm{g} \mathrm{m}^{-2}\right)$ no SimulArroz foi calculada por: Parcial $=$ Pesol $\times$ TotalG e TotalG $=$ Parcial / (Pmáx $(0,25))$ em que Parcial é o peso parcial de grãos $\left(\mathrm{g} \mathrm{m}^{-2}\right)$, Pesol é o peso de 1 grão $(\mathrm{g})$, TotalG é o número de grãos (grãos $\mathrm{m}^{-2}$ ) e Pmáx é o peso 


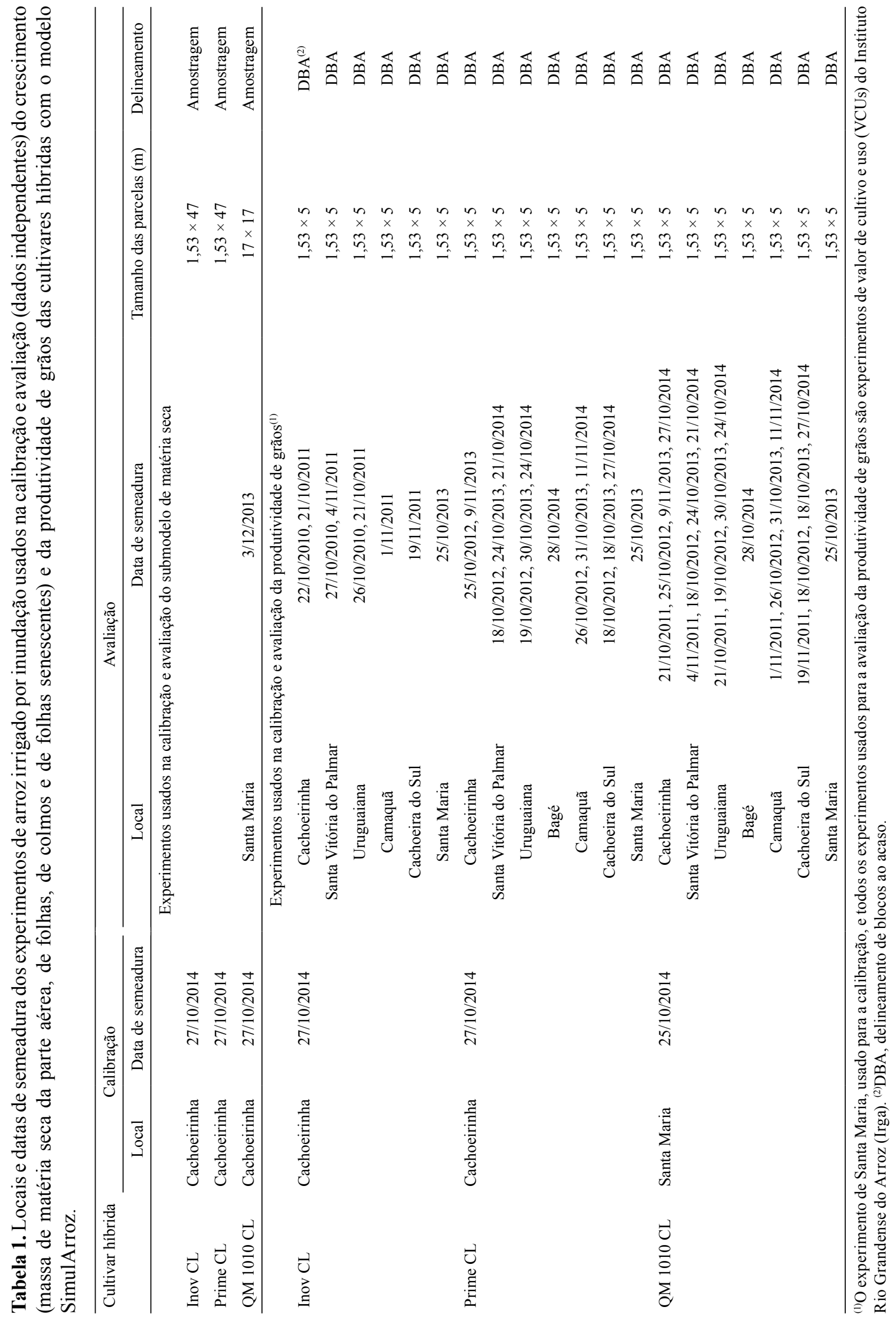


máximo de 1 grão $(\mathrm{g})$. Posteriormente a produtividade foi convertida para $\mathrm{Mg} \mathrm{ha}^{-1}$.

A introdução das três cultivares híbridas no modelo SimulArroz foi realizada por meio da calibração dos coeficientes genéticos nos processos que comandam o crescimento (equações 2, 3 e 4) e a produtividade de grãos (equações 5 e 6) com uso dos dados dos experimentos apresentados na Tabela 1. A calibração consistiu em estimar o valor de EUR para ajustar a MS total da parte aérea e as frações da tabela de contingência, uma vez que a fração destinada à parte aérea é então distribuída entre os diferentes órgãos da planta (folhas, colmos e panículas) de acordo com o estádio em que a cultura se encontra. $\mathrm{O}$ método utilizado para fazer este ajuste também foi o de aumento e diminuição de um valor inicial em 1\% para minimizar o erro (RQME) da estimativa (Samboranha et al., 2013).

A avaliação do modelo na simulação da massa de matéria seca de folhas, de colmos, de folhas senescentes e de produtividade de grãos foi realizada com dados independentes (Tabela 1). Para avaliar a produtividade, o SimulArroz foi rodado nos níveis tecnológicos "potencial" e "alto" com o objetivo de verificar qual nível tecnológico do SimulArroz mais se aproxima do manejo realizado nos VCUs. O nível potencial representa áreas experimentais sem estresse biótico e abiótico, enquanto o nível tecnológico alto representa lavouras manejadas segundo o projeto 10 do Irga, que preconiza o manejo integrado de plantas daninhas, insetos e doenças, adubação elevada e antecipação da época de semeadura. Os dados meteorológicos para rodar o modelo foram das estações automáticas do Instituto Nacional de Meteorologia localizadas o mais próximo possível de cada local.

O desempenho do modelo SimulArroz foi avaliado pelas estatísticas raiz do erro médio quadrático (RQME) e raiz do erro médio quadrático normalizado (RQMEn) conforme Janssen \& Heuberger (1995), índice "Bias", índice de concordância (dw), coeficiente de correlação de Pearson (r) segundo Samboranha et al. (2013), e coeficiente de eficiência de Nash-Sutcliffe (COE) conforme Nash \& Sutcliffe (1970).

O RQME expressa o erro médio do modelo (quanto menor o RQME, melhor é o modelo) e é calculado segundo Janssen \& Heuberger (1995):

$$
\mathrm{RQME}=\left[\left(\sum(\mathrm{Si}-\mathrm{Oi})^{2}\right) / \mathrm{n}\right]^{0,5}
$$

em que Si são os valores simulados, Oi são os valores observados e n é o número de observações.

O RQME normalizado foi calculado segundo Janssen \& Heuberger (1995):

$$
\mathrm{RQMEn}=100 \times \mathrm{RQME} / \overline{\mathrm{O}}
$$

em que $\overline{\mathrm{O}}$ é a média dos valores observados.

O valor de "Bias" foi calculado segundo Samboranha et al. (2013):

$$
\text { Bias }=\left(\sum \mathrm{Si}-\sum \mathrm{Oi}\right) /\left(\sum \mathrm{Oi}\right)
$$

O índice "dw" foi calculado segundo Borges \& Mendiondo (2007) e Samboranha et al. (2013):

$$
\mathrm{dw}=1-\left[\sum(\mathrm{Si}-\mathrm{Oi})^{2}\right] /[(|\mathrm{Si}-\overline{\mathrm{O}}|)+(|\mathrm{Oi}-\overline{\mathrm{O}}|)]^{2}
$$

O valor de $\mathrm{r}$ foi calculado segundo Borges \& Mendiondo (2007) e Samboranha et al. (2013):

$$
\mathrm{r}=\left[\sum(\mathrm{Oi}-\overline{\mathrm{O}}) \times(\mathrm{Si}-\mathrm{S})\right] /\left\{\left[\sum(\mathrm{Oi}-\overline{\mathrm{O}})^{2}\right]\left[\sum(\mathrm{Si}-\mathrm{S})^{2}\right]\right\}^{0,5}
$$

O valor de COE foi calculado segundo Nash \& Sutcliffe (1970):

$$
\mathrm{COE}=1-\sum(\mathrm{Si}-\mathrm{Oi})^{2} / \sum(\mathrm{Si}-\overline{\mathrm{O}})^{2}
$$

em que Si são os valores simulados, S é a média dos valores simulados, Oi são os valores observados, e Ō é a média dos valores observados.

\section{Resultados e Discussão}

Os valores calibrados de eficiência do uso da radiação (EUR), fator de formação de espiguetas (SOCF) e o peso máximo de um grão (Pmáx) para as três cultivares híbridas (Tabela 2) foram maiores do que os das cultivares convencionais $\left(2,39 \mathrm{~g} \mathrm{MJ}^{-1}, 65\right.$ espiguetas $\mathrm{g} \mathrm{MS}^{-1}$ e $0,024 \mathrm{~g}$ para EUR, SOCF e Pmáx, respectivamente) que constam no modelo SimulArroz (Rosa et al., 2015). As cultivares híbridas alocaram mais fotoassimilados, da parte aérea, nos colmos, ou seja, os colmos são grandes drenos, com translocação de $60 \%$ até o estádio de desenvolvimento (DVS) 0,43 (Tabela 3). Os colmos maiores são importantes para sustentar a maior panícula das cultivares híbridas (Coimbra et al., 2006).

A cultivar híbrida QM 1010 CL diferiu da convencional Irga 424 na partição de assimilados por apresentar maior produção de MS total da parte aérea e de colmos, e menor produção de MS de folhas

Pesq. agropec. bras., Brasília, v.51, n.12, p.1907-1917, dez. 2016 DOI: $10.1590 / \mathrm{S} 0100-204 X 2016001200001$ 
e de folhas senescentes (Figura 1). A simulação da MS total da parte aérea, de folhas, de colmos e de folhas senescentes da 'QM 1010 CL' apresentou um valor de raiz do erro médio quadrático (RQME) que variou de 28,3 a $113,2 \mathrm{~g} \mathrm{~m}^{-2} \mathrm{e}$ de raiz do erro médio quadrático normalizado (RQMEn) que variou de $13,6 \%$ a $50,8 \%$, enquanto o RQME para a 'Irga 424 ' variou de 57,0 a 265,7 $\mathrm{g} \mathrm{m}^{-2}$. Os valores do índice Bias são indicativos de uma superestimativa do modelo (4 a 28\%), para a cultivar híbrida QM 1010 CL e a convencional, exceto para a MS total da parte aérea e MS de folhas senescentes. $O$ índice dw e o coeficiente de correlação são indicativos do melhor desempenho do modelo, pois dw e $\mathrm{r}$ apresentaram valores acima de 0,95 , e o coeficiente de eficiência foi indicativo do bom ajuste do modelo com os dados observados (de 0,73 a 0,98), principalmente nas cultivares híbridas. Quanto à produtividade de grãos, o RQME e o RQMEn foram, respectivamente,

Tabela 2. Parâmetros do modelo SimulArroz eficiência do uso da radiação (EUR), fator de formação das espiguetas (SOCF, espiguetas $\mathrm{g}^{-1}$ de MS) e peso máximo de um grão (Pmáx), calibrados para as três cultivares de arroz híbrido introduzidas no modelo.

\begin{tabular}{lccc}
\hline Parâmetro & \multicolumn{3}{c}{ Cultivar de arroz híbrido } \\
\cline { 2 - 4 } & QM 1010 CL & Inov CL & Prime CL \\
\hline EUR $\left(\mathrm{g} \mathrm{MJ}^{-1}\right)$ & 2,93 & 2,75 & 2,65 \\
SOCF & 49 & 50 & 39 \\
Pmáx $(\mathrm{g})$ & 0,031 & 0,031 & 0,030 \\
\hline
\end{tabular}

2,0 $\mathrm{Mg}$ ha $^{-1}$ e $13,6 \%$ para a 'QM $1010 \mathrm{CL}^{\prime}$ e $0,9 \mathrm{Mg} \mathrm{ha}^{-1}$ e $8,7 \%$ para o 'Irga 424', mas não foi possível calcular as outras estatísticas, pois só há um valor simulado $\times$ observado (Figura 1E).

Os erros nas simulações da matéria seca e da produtividade de grãos obtidos neste trabalho são próximos de outros trabalhos que utilizaram o modelo Oryza2000 (Bouman et al., 2004) (Tabela 4 e Figura 1). Ao ser utilizado o modelo no nível tecnológico potencial, o RQME variou de 2,4 a 5,0 $\mathrm{Mg} \mathrm{ha}^{-1}$, e o RQMEn de 23,5 a 46,5\% (Figura 2A), valores próximos da variação de RQME e RQMEn da cultivar convencional Irga 424. Porém, quando o modelo foi utilizado para o nível tecnológico alto, os valores do RQME foram menores, tendo variado de 2,0 a $2,6 \mathrm{Mg} \mathrm{ha}^{-1}$, e de $19,0 \%$ a $23,7 \%$ quanto ao RQMEn. Na China, Boling et al. (2011) reportaram para a cultura do arroz um RQME de $0,6 \mathrm{Mg} \mathrm{ha}^{-1} \mathrm{e}$ um RQMEn de $20,4 \%$, ou seja, os erros do presente estudo estão dentro da faixa reportada pelos autores referidos.

$\mathrm{O}$ vigor híbrido calculado com base nos dados simulados de produtividade de grãos foi de $28 \%$ na condição "potencial" do modelo e $18 \%$ no nível tecnológico "alto" (Figura 2). Esses dois valores estão de acordo com os relatados na literatura, em que as cultivares híbridas podem alcançar produtividades 20 a $30 \%$ maiores do que as convencionais (Coimbra et al., 2006; Fu et al., 2015; Shi et al., 2015). O vigor híbrido calculado para os dados de produtividade nos VCUs do Irga foi de 13\%, o que indica que a condução dos experimentos nos VCUs se enquadrou melhor no nível tecnológico "alto" do modelo SimulArroz. Com

Tabela 3. Parâmetros da partição de matéria seca, estádio de desenvolvimento da cultura do arroz irrigado (DVS), folhas $(\mathrm{F})$, colmos (C) e grãos (G), do modelo SimulArroz calibrados para três híbridos de arroz.

\begin{tabular}{|c|c|c|c|c|c|c|}
\hline \multirow[t]{3}{*}{$\mathrm{DVS}^{(1)}$} & \multirow[t]{3}{*}{ Parte aérea } & \multicolumn{4}{|c|}{ Híbridos } & \multirow{3}{*}{$\begin{array}{c}\text { Convencional } \\
\text { Irga } 424 \\
\text { F; C; G }\end{array}$} \\
\hline & & QM $1010 \mathrm{CL}$ & Inov CL & Prime CL & Média $^{(2)}$ & \\
\hline & & $\mathrm{F} ; \mathrm{C} ; \mathrm{G}$ & $\mathrm{F} ; \mathrm{C} ; \mathrm{G}$ & $\mathrm{F} ; \mathrm{C} ; \mathrm{G}$ & $\mathrm{F} ; \mathrm{C} ; \mathrm{G}$ & \\
\hline 0 & 0,50 & 0,$5 ; 0,5 ; 0,0$ & 0,$5 ; 0,5 ; 0,0$ & 0,$3 ; 0,7 ; 0,0$ & 0,$4 ; 0,6 ; 0,0$ & 0,$6 ; 0,4 ; 0,0$ \\
\hline 0,43 & 0,75 & 0,$5 ; 0,5 ; 0,0$ & 0,$5 ; 0,5 ; 0,0$ & 0,$3 ; 0,7 ; 0,0$ & 0,$4 ; 0,6 ; 0,0$ & 0,$6 ; 0,4 ; 0,0$ \\
\hline 0,5 & 0,75 & 0,$2 ; 0,8 ; 0,0$ & 0,$2 ; 0,8 ; 0,0$ & 0,$6 ; 0,4 ; 0,0$ & 0,$3 ; 0,7 ; 0,0$ & 0,$3 ; 0,7 ; 0,0$ \\
\hline 0,75 & 0,75 & 0,$0 ; 0,3 ; 0,7$ & 0,$3 ; 0,7 ; 0,0$ & 0,$0 ; 0,0 ; 1,0$ & 0,$1 ; 0,3 ; 0,6$ & 0,$4 ; 0,6 ; 0,0$ \\
\hline 1,0 & 1,0 & 0,$0 ; 0,0 ; 1,0$ & 0,$0 ; 0,0 ; 1,0$ & 0,$0 ; 0,0 ; 1,0$ & 0,$0 ; 0,0 ; 1,0$ & 0,$0 ; 0,0 ; 1,0$ \\
\hline 1,2 & 1,0 & 0,$0 ; 0,0 ; 1,0$ & 0,$0 ; 0,0 ; 1,0$ & 0,$0 ; 0,0 ; 1,0$ & 0,$0 ; 0,0 ; 1,0$ & 0,$0 ; 0,0 ; 1,0$ \\
\hline 1,6 & 1,0 & 0,$0 ; 0,0 ; 1,0$ & 0,$0 ; 0,0 ; 1,0$ & 0,$0 ; 0,0 ; 1,0$ & 0,$0 ; 0,0 ; 1,0$ & 0,$0 ; 0,0 ; 1,0$ \\
\hline 2,0 & 1,0 & 0,$0 ; 0,0 ; 1,0$ & 0,$0 ; 0,0 ; 1,0$ & 0,$0 ; 0,0 ; 1,0$ & 0,$0 ; 0,0 ; 1,0$ & 0,$0 ; 0,0 ; 1,0$ \\
\hline
\end{tabular}

${ }^{(1)}$ O DVS é 0 na emergência, 0,65 na diferenciação da panícula, 1,0 na antese e 2,0 na maturidade fisiológica. ${ }^{(2)}$ Média dos três híbridos. 
a maioria das estatísticas, o melhor desempenho do modelo SimulArroz em simular a produtividade nos VCUs também foi obtido quando o modelo foi rodado no nível tecnológico "alto" (Tabela 5). Isso acontece em razão da dificuldade de conduzir experimentos de campo na condição "potencial", pois o potencial de produtividade é estimado por modelos que assumem o perfeito manejo da água e nutrientes e ausência de
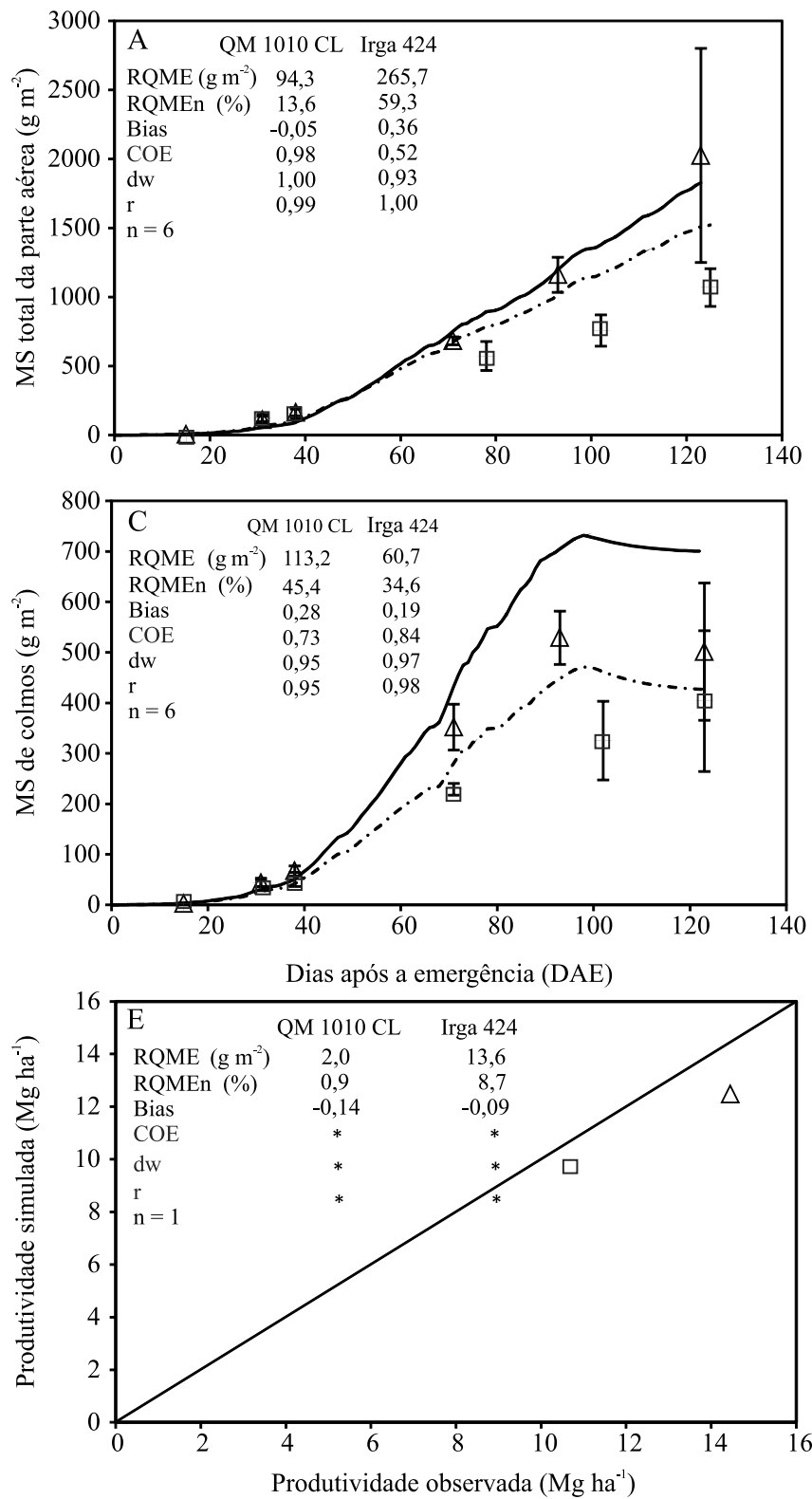

estresse biótico, o que em experimentos de campo é praticamente impossível de ser alcançado (Ittersum et al., 2013).

A produtividade em todas as datas de semeadura e locais foi maior na cultivar QM $1010 \mathrm{CL}$, seguida de 'Inov CL', 'Prime CL' e 'Irga 424' (Figura 3 A e B), tendo sido consistente com a produtividade esperada para o grupo de maturação das cultivares híbridas QM 1010
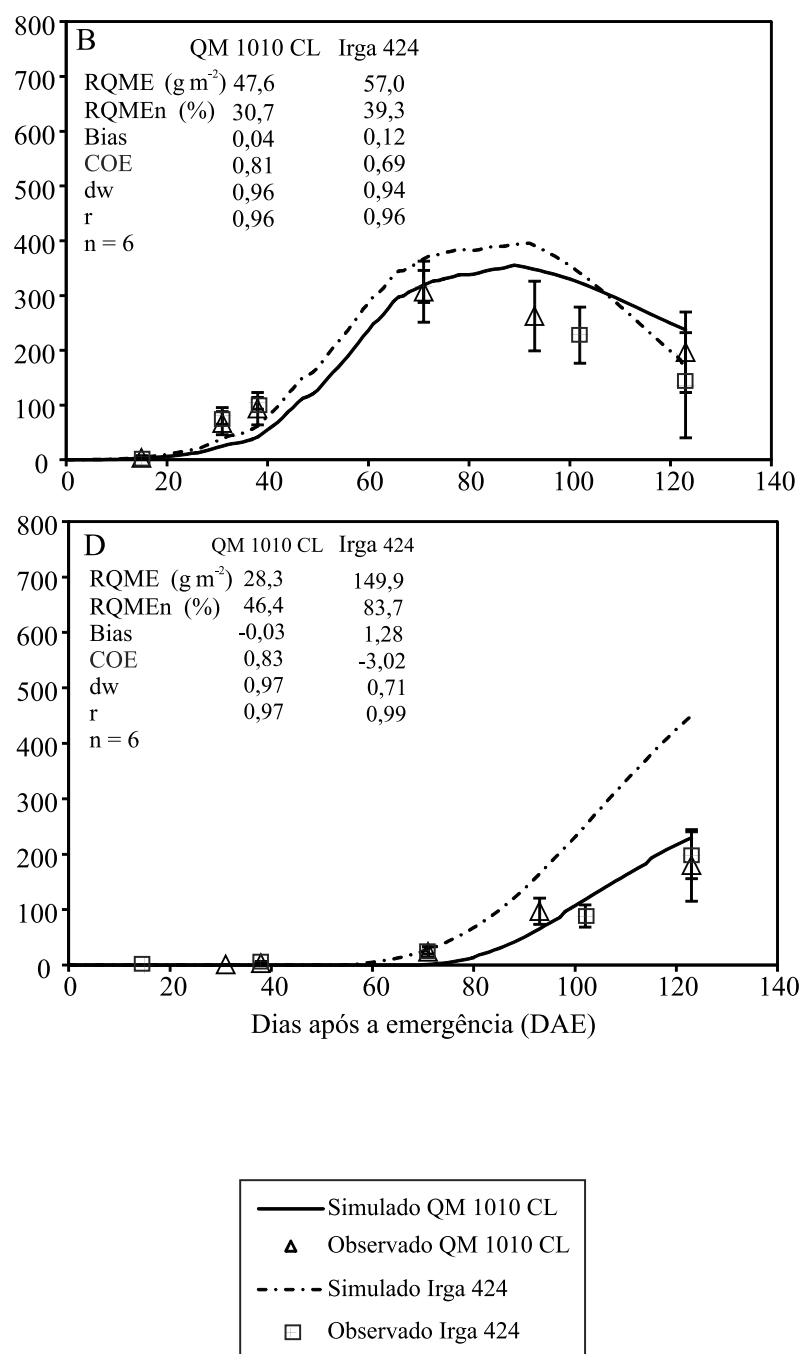

Figura 1. Massa de matéria seca (MS) total da parte aérea (A), MS de folhas (B), MS de colmos (C), MS de folhas senescentes (D) e produtividade de grãos a 13\% de umidade (E) observada e simulada com o modelo SimulArroz para a cultivar híbrida QM 1010 CL e para a cultivar convencional Irga 424 de arroz irrigado no ano agrícola 2013/2014 em Santa Maria, Rio Grande do Sul. As barras são os desvios-padrões observados. 
CL (de ciclo médio), Inov CL e Prime CL (ambos de ciclo precoce) e com o vigor expresso pelas cultivares híbridas (28\%) em comparação com a cultivar convencional Irga 424 (ciclo médio). A variação de produtividade de grãos entre datas de semeadura também foi verificada por Rosa et al. (2015), pois o potencial de produtividade diminui com o atraso da data de semeadura.

Entre os locais (Figura 3C), os maiores potenciais de produtividade na Fronteira Oeste e Campanha (representadas pelos municípios de Uruguaiana e Bagé, respectivamente) são explicados pela maior disponibilidade de radiação solar nestas regiões (Steinmetz et al., 2013).

A produtividade de arroz no Rio Grande do Sul também pode variar entre anos, com destaque para o ano agrícola 2011/12, em que se obteve a maior produtividade simulada pelo modelo (Figura 3D). Este ano foi de "La Niña", com precipitação e umidade relativa do ar abaixo da normal climatológica e

Tabela 4. Estatísticas RQME (Raiz do quadrado médio do erro) e RQMEn (Raiz do quadrado médio do erro normalizada) da simulação dos compartimentos da matéria seca (MS) do arroz híbrido com o modelo SimulArroz obtidos neste estudo e com o modelo Oryza2000 descritos na literatura.

\begin{tabular}{|c|c|c|c|c|}
\hline Parâmetros de crescimento & $\operatorname{RQME}\left(\mathrm{g} \mathrm{m}^{-2}\right)$ & RQMEn (\%) & Modelo & Local e Fonte \\
\hline \multirow[t]{3}{*}{ MS da parte aérea } & 57,0 & 20,0 & Oryza2000 & Filipinas, Boling et al. (2011) \\
\hline & 216,1 & 19,0 & Oryza2000 & Chile, Artacho et al. (2011) \\
\hline & 94,3 & 13,6 & SimulArroz & Rio Grande do Sul, Brasil, Rosa et al. (2015) \\
\hline \multirow[t]{2}{*}{ MS de folhas } & 227,3 & 37,0 & Oryza2000 & Chile Artacho et al. (2011) \\
\hline & 47,6 & 30,7 & SimulArroz & Rio Grande do Sul, Brasil, Rosa et al. (2015) \\
\hline \multirow[t]{2}{*}{ MS de colmos } & 90,2 & 47,0 & Oryza2000 & Chile, Artacho et al. (2011) \\
\hline & 113,2 & 45,4 & SimulArroz & Rio Grande do Sul, Brasil, Rosa et al. (2015) \\
\hline \multirow[t]{2}{*}{ MS de folhas senescentes } & 41,7 & 38,0 & Oryza2000 & Chile, Artacho et al. (2011) \\
\hline & 28,3 & 46,4 & SimulArroz & Rio Grande do Sul, Brasil, Rosa et al. (2015) \\
\hline \multirow[t]{2}{*}{ Produtividade } & - & 19,0 & Oryza2000 & Chile, Artacho et al. (2011) \\
\hline & 2,0 & 13,0 & SimulArroz & Rio Grande do Sul, Brasil, Rosa et al. (2015) \\
\hline
\end{tabular}
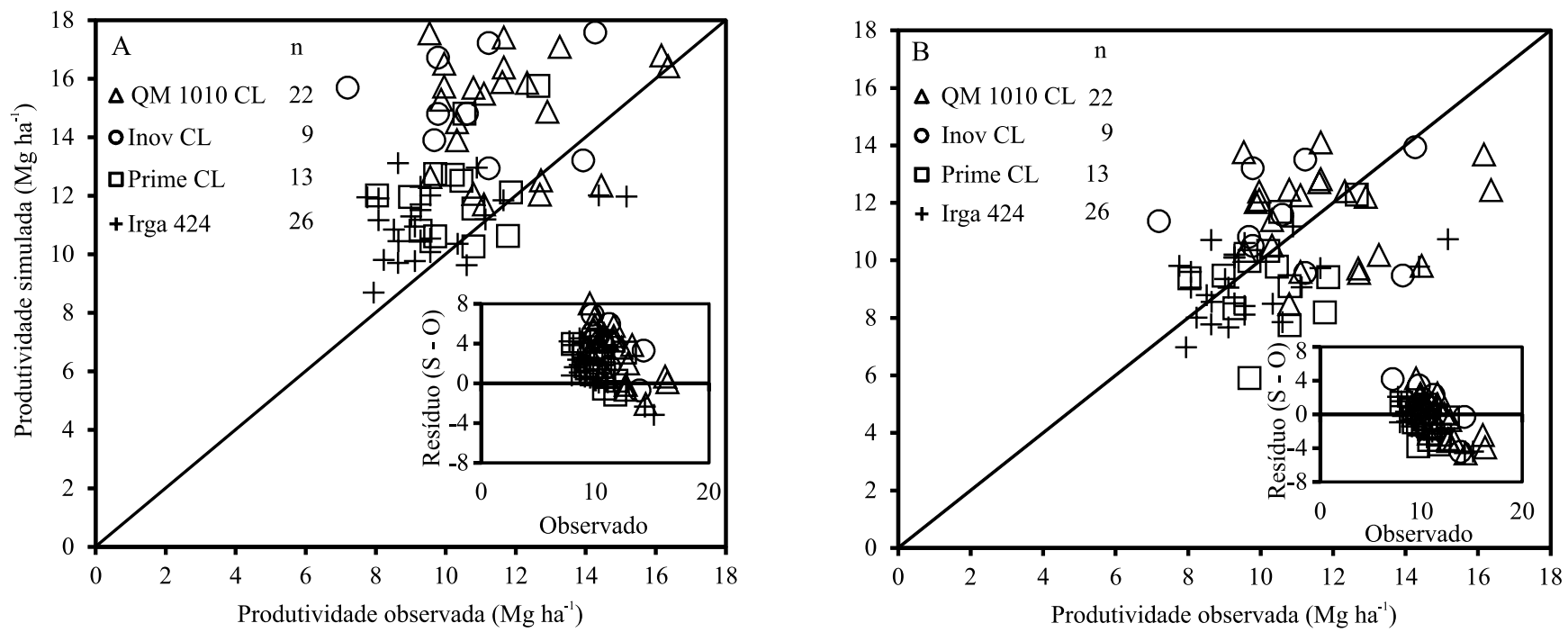

Figura 2. Produtividade de grãos a $13 \%$ de umidade observada nos experimentos Valor de Cultivo e Uso (VCUs) do Instituto Rio Grandense do Arroz (Irga) e simulada pelo modelo SimulArroz das cultivares híbridas QM 1010 CL, Inov CL e Prime CL e da cultivar convencional Irga 424 de arroz, em sete locais (Cachoeirinha, Santa Vitória do Palmar, Uruguaiana, Bagé, Camaquã, Cachoeira do Sul e Santa Maria), em 5 anos agrícolas (2010/2011, 2011/2012, 2012/2013, 2013/2014 e 2014/2015). Os dados simulados são no nível potencial (A) e alto (B) do SimulArroz. $\mathrm{n}$ = número de observações. 
Tabela 5. Estatísticas do desempenho do modelo SimulArroz na simulação da produtividade de grãos a 13\% de umidade de três híbridos e uma cultivar convencional (Irga 424) de arroz irrigado em sete locais (Cachoeirinha, Santa Vitória do Palmar, Uruguaiana, Bagé, Camaquã, Cachoeira do Sul e Santa Maria) do Rio Grande do Sul, em 5 anos agrícolas (2010/2011, 2011/2012, 2012/2013, 2013/2014 e 2014/2015).

\begin{tabular}{|c|c|c|c|c|c|}
\hline \multirow{2}{*}{$\begin{array}{l}\text { Nível tecnológico } \\
\text { no SimulArroz }\end{array}$} & \multirow[t]{2}{*}{ Estatística $^{(1)}$} & \multicolumn{3}{|c|}{ Híbridos } & \multirow[t]{2}{*}{ Irga 424} \\
\hline & & QM $1010 \mathrm{CL}$ & Inov CL & Prime CL & \\
\hline \multirow{6}{*}{ Potencial } & RQME & 4,1 & 5,0 & 2,4 & 2,3 \\
\hline & RQMEn & 34,5 & 46,5 & 23,5 & 23,3 \\
\hline & $\mathrm{dw}$ & 0,40 & 0,37 & 0,46 & 0,52 \\
\hline & $\mathrm{COE}$ & $-3,52$ & $-4,96$ & $-2,99$ & $-0,63$ \\
\hline & Bias & 0,27 & 0,40 & 0,17 & 0,14 \\
\hline & $\mathrm{r}$ & 0,08 & 0,02 & 0,33 & 0,29 \\
\hline \multirow{6}{*}{ Alto } & RQME & 2,4 & 2,6 & 2,0 & 1,8 \\
\hline & RQMEn & 20,4 & 23,7 & 19,0 & 18,5 \\
\hline & $\mathrm{dw}$ & 0,42 & 0,44 & 0,52 & 0,53 \\
\hline & $\mathrm{COE}$ & $-0,58$ & $-0,55$ & $-1,62$ & $-0,02$ \\
\hline & Bias & $-0,02$ & 0,06 & $-0,10$ & $-0,05$ \\
\hline & $\mathrm{r}$ & 0,04 & 0,09 & 0,30 & 0,36 \\
\hline
\end{tabular}

(1)RQME, raiz do quadrado médio do erro ( $\left.\mathrm{Mg} \mathrm{ha}^{-1}\right)$; RQMEn, raiz do quadrado médio do erro normalizada (\%); dw, índice de concordância; COE, coeficiente de eficiência de Nash-Sutcliffe; Bias, índice Bias; r, coeficiente de correlação de Pearson.
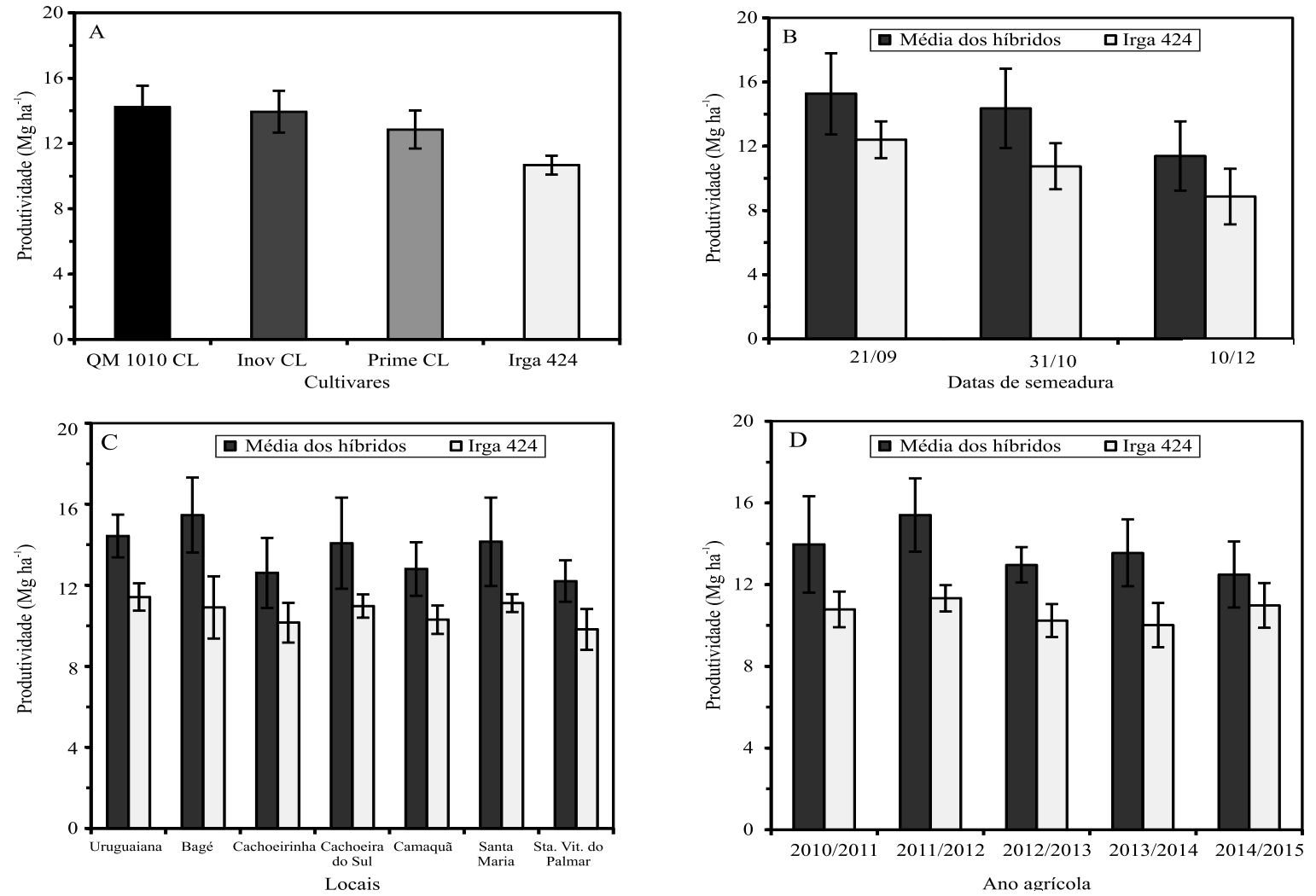

Figura 3. O efeito de cultivar (A), de datas de semeadura (B), de local (C) e de ano agrícola (D) sobre a produtividade de grãos a 13\% de umidade das cultivares híbridas QM 1010 CL, Inov CL e Prime CL e da cultivar convencional Irga 424 de arroz em sete locais do Rio Grande do Sul (Cachoeirinha, Vitória do Palmar, Uruguaiana, Bagé, Camaquã, Cachoeira do Sul, Santa e Santa Maria), para as datas de semeadura de 21/9, 31/10 e 10/12 em cinco anos agrícolas (2010/2011, 2011/2012, 2012/2013, 2013/2014 e 2014/2015). 
radiação solar maior, o que resultou em maior crescimento e produtividade, pois a disponibilidade de radiação solar na fase reprodutiva (da diferenciação da panícula até a antese) e durante o enchimento de grãos é o principal elemento meteorológico que determina a produtividade potencial em arroz.

\section{Conclusões}

1. A calibração do modelo SimulArroz utilizada é eficiente em simular o acúmulo de matéria seca e a produtividade de grãos de cultivares de arroz híbrido nas regiões orizícolas irrigadas do Rio Grande do Sul.

2. Nas cultivares híbridas de arroz são alocados mais fotoassimilados da parte aérea nos colmos, para sustentar a maior panícula, em comparação com cultivares convencionais.

3. O modelo SimulArroz tem melhor desempenho em simular o acúmulo de matéria seca e a produtividade das cultivares de arroz irrigado do Rio Grande do sul nos VCUs, quando rodado na condição de nível tecnológico "alto".

\section{Agradecimentos}

Ao Conselho Nacional de Desenvolvimento Científico e Tecnológico (CNPq) e ao Instituto Rio Grandense do Arroz (Irga), pelo apoio financeiro.

\section{Referências}

AGGARWAL, P.K.; KALRA, N.; CHANDER, S.; PATHAK, H. InfoCrop: a dynamic simulation model for the assessment of crop yields, losses due to pests, and environmental impact of agro-ecosystems in tropical environments. I. Model description. Agricultural Systems, v.89, p.1-25, 2006. DOI: 10.1016/j. agsy.2005.08.001.

ARTACHO, P.; MEZA, F.; ALCALDE, J.A. Evaluation of the Oryza2000 rice growth model under nitrogen-limited conditions in an irrigated Mediterranean environment. Chilean Journal of Agricultural Research, v.71 p.23-33, 2011. DOI: 10.4067/S071858392011000100003.

BOLING, A.A.; BOUMAN, B.A.M.; TUONG, T.P.; KONBOON, Y.; HARNPICHITVITAYA, D. Yield gap analysis and the effect of nitrogen and water on photoperiod-sensitive Jasmine rice in north-east Thailand. Wageningen Journal of Life Science, v.58, p.11-19, 2011. DOI: 10.1016/j.njas.2010.05.001.

BORGES, A.C. de; MENDIONDO, E.M. Comparação entre equações empíricas para estimativa da evapotranspiração de referência na Bacia do Rio Jacupiranga. Revista Brasileira de Engenharia Agrícola e Ambiental, v.11, p.293-300, 2007.
BOUMAN, B.A.M.; KROPFF, M.J.; TUONG, T.P.; WOPEREIS, M.C.S.; TEN BERGE, H.F.M.; LAAR, H.H. van. Oryza 2000: modeling lowland rice. Version 2.12. Los Baños: International Rice Research Institute, 2004. Disponível em: <http://www. knowledgebank.irri.org/oryza2000/default.htm>. Acesso em: 20 maio 2015.

CHIN, J.H.; CHU, S.-H.; JIANG, W.; CHO, Y.-I.; BASYIRIN, R.; BRAR, D.S.; KOH, H.-J. Identification of QTLs for hybrid fertility in inter-subspecific crosses of rice (Oryza sativa L.). Genes and Genomics, v.33, p.39-48, 2011. DOI: 10.1007/s13258-010-0100-z.

COIMBRA, J.L.M.; OLIVEIRA, A.C.de; CARVALHO, F.I.F. de; MAGALHÃES JÚNIOR, A.M. de; FAGUNDES, P.R.R.; KOPP, M.M. Heterose em arroz híbrido. Revista Brasileira de Agrociência, v.12, p.257-264, 2006.

CONAB. Companhia Nacional de Abastecimento. Séries históricas. Disponível em: < http://www.conab.gov.br/conteudos. php?a=1252\&>. Acesso em: 18 jan. 2016.

COUNCE, P.A.; KEISLING, T.C.; MITCHELL, A.J. A uniform, objective, and adaptive system for expressing rice development, Crop Science, v.40, p.436-443, 2000. DOI: 10.2135/cropsci2000.402436x.

DINGKUHN, M.; LAZA, M.R.C.; KUMAR, U.; MENDEZ, K.S.; COLLARD, B.; JAGADISH, K.; SINGH, R.K.; PADOLINA, T.; MALABAYABAS, M.; TORRES, E.; REBOLLEDO, M.C.; MANNEH, B., SOW, A. Improving yield potential of tropical rice: achieved levels and perspectives through improved ideotypes. Field Crops Research, v.182, p.43-59, 2015. DOI: 10.1016/j.fcr.2015.05.025.

ESTEFANEL, V.; SCHNEIDER, F.M.; BERLATO, M.A.; BURIOL, G.A.; HELDWEIN, A.B. Insolação e radiação solar na região de Santa Maria, RS: I - estimativa da radiação solar global incidente a partir dos dados de insolação. Revista Centro de Ciências Rurais, v.20, p.203-218, 1990.

FU, G.; ZHANG, C.; YANG, Y.; XIONG, J.; YANG, X.; ZHANG, X.; JIN, Q.; TAO, L. Male parent plays a more important role in heat tolerance in three-line hybrid rice. Rice Science, v.22, p.116-122, 2015. DOI: 10.1016/j.rsci.2015.05.015.

GABRIEL, L.F.; STRECK, N.A.; ROBERTI, D.R.; CHIELLE, Z.G.; UHLMANN, L.O.; SILVA, M.R. da.; SILVA, S.D. da. Simulating cassava growth and yield under potential. Agronomy Journal, v.106, p.1119-1137, 2014. DOI: 10.2134/agronj2013.0187.

GRASSINI, P.; TORRION, J.A.; YANG, H.S.; REES, J.; ANDERSEN, D.; CASSMAN, K.G.; SPECHT, J.E. Soybean yield gaps and water productivity in the western U.S. Corn Belt. Field Crops Research, v.179, p.150-163, 2015. DOI: 10.1016/j. fcr.2015.04.015.

IRGA. Instituto Rio Grandense do Arroz. Evolução da Semeadura - Safra 2015/16. Disponível em: <http:// www.irga.rs.gov.br/upload/20160129171028evolucao_da_ semeadura_15_16.pdf $>$. Acesso em: 25 jan. 2016a.

IRGA. Instituto Rio Grandense do Arroz. Série Histórica de Produção e Produtividade - RS x BR. Disponível em: <http:// www.irga.rs.gov.br/upload/20150720134318producao_rs_e_ brasil.pdf $>$. Acesso em: 25 jan. 2016 b.

IRGA. Instituto Rio Grandense do Arroz. [Levantamento da área semeada com arroz irrigado híbrido no Rio Grande 
do Sul, 2015]. Disponível em: <http://www.irga.rs.gov.br/ upload/20160408161314cultivares_rs_2015_16.pdf>. Acesso em: 20 set. 2015.

ITTERSUM, M.K. van; CASSMAN, K.G.; GRASSINI, P.; WOLF, J.; TITTONELL; P.; HOCHMAN, Z. Yield gap analysis with local to global relevance - a review. Field Crops Research, v.143, p.4-17, 2013. DOI: 10.1016/j.fcr.2012.09.009.

JANSSEN, P.H.M.; HEUBERGER, P.S.C. Calibration of processoriented models. Ecological Modelling, v.83, p.55-56, 1995. DOI: 10.1016/0304-3800(95)00084-9.

KINIRY, J.R.; MCCAULEY, G.; XIE Y.; ARNOLD, J.G. Rice parameters describing crop performance of four U.S. cultivars. Agronomy Journal, v.93, p.1354-1361, 2001.

LI, T.; RAMAN, A.K.; MARCAIDA III, M.; KUMAR, A.; ANGELES, O.; RADANIELSON, A.M. Simulation of genotype performances across a larger number of environments for rice breeding using Oryza2000. Field Crops Research, v.149, p.312321, 2013. DOI: 10.1016/j.fcr.2013.05.006.

MOTA, F.S. da. Disponibilidade da radiação solar e risco de frio no período reprodutivo do arroz irrigado em diferentes regiões do Rio Grande do Sul. Lavoura Arrozeira, v.48, p.8-10, 1995.

NASH, J.E.; SUTCLIFFE, J.V. River flow forecasting through conceptual models part I - a discussion of principles. Journal of Hydrology, v.10, p.282-290, 1970. DOI: 10.1016/0022-1694 (70)90255-6.

REUNIÃO TÉCNICA DA CULTURA DO ARROZ IRRIGADO, 30., 2014, Bento Gonçalves. Arroz irrigado: recomendações técnicas da pesquisa para o sul do Brasil. Santa Maria: Sosbai, 2014. 189 p.

ROSA, H.T.; WALTER, L.C.; STRECK, N.A.; CARLI, C. de; RIBAS, G.G.; MARCHESAN, E. Simulação do crescimento e produtividade de arroz no Rio Grande do Sul pelo modelo SimulArroz. Revista Brasileira de Engenharia Agrícola e Ambiental, v.19, p.1159-1165, 2015. DOI: 10.1590/1807-1929/ agriambi.v19n12p1159-1165.

SAMBORANHA, F.K.; STRECK, N.A.; UHLMANN, L.O.; GABRIEL, L.F. Modelagem matemática do desenvolvimento foliar em mandioca. Revista Ciência Agronômica, v.44, p.815-824, 2013. DOI: 10.1590/S1806-66902013000400019.
SENTELHAS, P.C.; BATTISTI, R.; CÂMARA, G.M.S.; FARIAS, J.R.B.; HAMPF, A.C.; NENDEL, C. The soybean yield gap in Brazil-magnitude, causes and possible solutions for sustainable production. Journal of Agricultural Science, v.153, p.1394-1411, 2015. DOI: 10.1017/S0021859615000313.

SHI, W.; YIN, X.; STRUIK, P.C.; XIE, F.; SCHMIDT, R.C.; JAGADISH, K.S.V. Grain yield and quality responses of tropical hybrid rice to high night-time temperature. Field Crops Research, v.190, p.18-25, 2015. DOI: 10.1016/j. fcr.2015.10.006.

SOLTANI, A.; ZEINALI, E.; GALESHI, S.; NIARI, N. Simulating GFDL predicted climate change impacts on rice cropping in Iran. Journal of Agricultural Science and Technology, v.3, p.81-90, 2001.

STEINMETZ, S.; DEIBLER, A.N.; SILVA, J.B. da. Estimativa da produtividade de arroz irrigado em função da radiação solar global e da temperatura minima do ar. Ciência Rural, v.43, p.206-211, 2013. DOI: 10.1590/S0103-84782013000200003.

STRECK, N.A.; LAGO, I.; OLIVEIRA, F.B.; HELDWEIN, A.B.; AVILA, L.A.de.; BOSCO, L.C. Modeling the development of cultivated rice and weedy red rice. Transactions of the ASABE, v.54, p.371-384, 2011. DOI: 10.13031/2013.36234.

STRECK, N.A.; ROSA, H.T.; WALTER, L.C.; SILVA, M.R. da; UHLMANN, L.O. CO2-response function of radiation use efficiency in rice for climate change scenarios. Pesquisa Agropecuária Brasileira, v.47, p.879-885, 2012. DOI: 10.1590/ S0100-204X2012000700001.

TORRION, J.; SETIYONO, T.D.; CASSMAN, K.; SPECHT, J. Soybean phenology simulation in the north-central United States. Agronomy Journal, v.103, p.1661-1667, 2011. DOI: 10.2134/agronj2011.0141.

USDA. United States Department of Agriculture. Production, Supply and Distribution Online. Disponível em: $<$ http:// www.usda.gov/wps/portal/usda/usdahome?navid=DATA STATISTICS>. Acesso em: 20 jan. 2016.

WALTER, L.C.; STRECK, N.A.; ROSA, H.T.; FERRAZ, S.E.T.; CERA, J.C. Mudanças climáticas e seus efeitos no rendimento de arroz irrigado no Rio Grande do Sul. Pesquisa Agropecuária Brasileira, v.49, p.915-924, 2014. DOI: 10.1590/S0100204X2014001200001.

Recebido em 8 de março de 2016 e aprovado em 6 de junho de 2016

Pesq. agropec. bras., Brasília, v.51, n.12, p.1907-1917, dez. 2016 DOI: 10.1590/S0100-204X2016001200001 